\title{
A Survey on Smart Monitoring System of Environment Based on IoT
}

\author{
Raghad Hazim AlSheikh* \\ raghad.h.alshekh@uomosul.edu.iq
}

\author{
Rabea Mwafaq Hagem* \\ rabeehagem@uomosul.edu.iq
}

Omer Salim**

omer.salim@monash.edu

\footnotetext{
*Computer Engineering Department, Collage of Engineering, University of Mosul **Clayton Campus, Monash University, Melbourne, Australia
}

Received: $13 / 11 / 2020$

Accepted: 20/1/2021

\begin{abstract}
:
This paper introduces different topics about Air pollution monitoring based on IoT (internet of things). It describes different methods used for monitoring and measuring Air pollution using different techniques. Some papers discussed Air pollution monitoring using (Arduino uno, Raspberry or ESP8266, PIC microcontroller). All would be presented to improve air monitoring efficiently and to clarify the tools used in each technique giving the differences between one technique and another. Reducing air pollution is necessary for the future, and cities around the world are using technology and smart city initiatives to take on the problem because it is considered as the major environmental risk factor for human health. Air pollution causes heart disease, stroke, chronic obstructive pulmonary disease, lung cancer and acute lower respiratory disease. Therefore, it is important to reduce traffic and output from factories to decrease pollution
\end{abstract}

Keywords:

IoT, Air pollution, Arduino, Raspberry, ESP8266, sensors, WSN (wireless sensor network).

https://rengj.mosuljournals.com

Email: alrafidain_engjournal1@uomosul.edu.iq

\section{Introduction :}

The Internet of Things (IoT): it is the third revolution in digital technology after the computer and the Internet, it is also based on the standard and interoperable communication protocols fueled by the leading technologies like Radio-Frequency Identification (RFID) and Wireless Sensor Networks (WSNs). It is based on IFTTT (IF THIS THEN THAT) which is a free web service for creating strings of simple conditional statements [1]. The architecture of IoT consists of three basic layers, the perception layer , the network layer, and the application layer as shown in Figure(1)[2].IoT aims to provide advanced and effective monitoring and control services and provide real-time data acquisition.

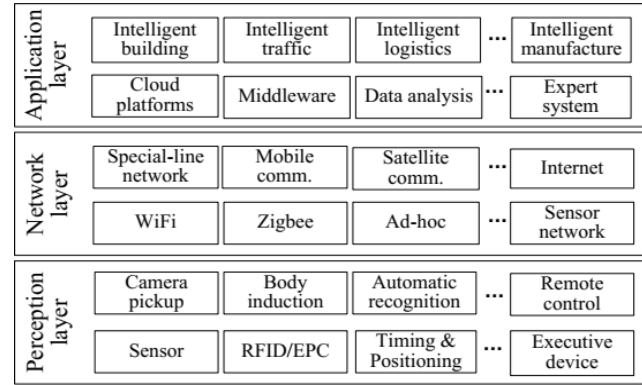

Figure (1) IoT architecture [3]

Pollution is divided into three main types: air pollution, water pollution, and soil pollution.

Air pollution refers to the release of pollutants into the air that are detrimental to human health and the planet as a whole, it comes from energy use production, burning fossil fuels releases gases and chemicals into the air.

Water pollution: is defined as adding harmful chemicals to water. Water pollution may come from industrial waste and runoff from mined areas [4]. 
Soil pollution is known as the existence of toxic chemicals in the soil in high concentrations enough to cause harm to human health and the ecosystem [5].

Wireless Sensor Networks (WSNs) are known as a self-configured and infrastructuredwireless networks to monitor environmental conditions such as temperature, sound, vibration, pressure, motion or pollutants and to pass data cooperatively through the network to a main position or sink where the data can be showed and analyzed [6]. WSN is shown in Figure (2).

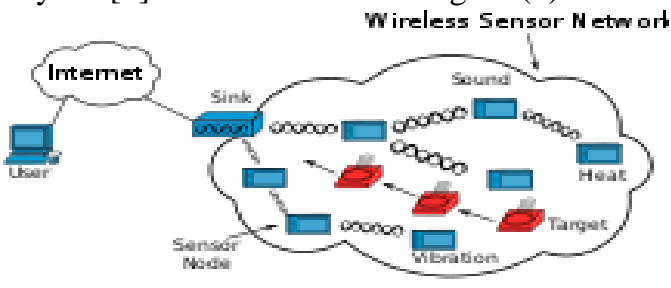

Figure (2) wireless sensor network

\section{Literature Review}

Srinivas Devarakonda and others provided Vehicle-based mobile phone to measure accurate real-time air quality.Two cost-effective models have been proposed for data cultivation, one for public transportation and the second for personal transportation. Six air pollutants have been measured which are: PM, ozone, carbon monoxide, sulfur oxides, Nitrogen oxides, and lead. They suggested a Mobile Sensor Box (MSB) that includes a controller board with more sensors, two terminals of the GPS receiver and a cellular modem that will provide the power supply needed to run the model [7].

Souvik Manna and others presented Air pollution system with a wireless sensor network which is considered as an effective use of IoT to handle vehicular pollution. Pollution can be collected on different city roads, and analyzed using WSN, Arduino Uno, RFID and MQ sensors to measure carbon monoxide, sulfur dioxide, nitrogen dioxide, and methane. Taking advantage of the Quality Index (AQI), and an algorithm will be implemented to gather data, bring out duplicates, filter and summarize worthless readings in a simpler form, and develop Mobile Discovery Net (MoDisNet) using GUSTO sensor technology to monitor and analyze pollution in real-time depending on traffic conditions, emissions, ambient pollutant concentration [8].

Shailendra Singh and others used WSN for greenhouse monitoring using YIKTIX hardware platform including humidity and temperature with the use of the Central Data Acquisition Unit (CDAU) to monitor indoor greenhouse conditions locally or remotely [9].

Muhammad Saqib Jamil and others also introduced WSN concept with the inclusion of the internet of things and LTE-M objects, and modules that are cheaper, and more efficient for M2M devices which may be useful for technologies for several other applications in making the smart cities [10].

Bedoui and others used WSN, and Zigbee under LABWIEW environment to measure hydrogen, sulfide gas, humidity and temperature the results show the effectiveness of this technique in terms of detection speed and real-time response with low-cost and low power because of using ZigBee with WSN the system includes three steps: first is data acquisition ,second is the wireless transmission and finally is data processing .To improve it stations can be replaced by a small portable metering system that includes various gas sensors[11].

The goal of Snehal Sirsikar and Priya Karemore review is shedding light on some of the techniques used to monitor air pollution and the effectiveness of these techniques, and identifying important research in this important field. Major gases that directly affect human health, carbon monoxide (CO), hydrogen sulfide, Sulfur dioxide (SO2), nitrogen dioxide (NO2).

Methods used:

1- Pollution monitoring technology: air pollution monitoring by uses of a group of GPRS sensors over the Internet

2- Wireless sensor network for real-time monitoring

3-Central monitoring: different sensors are deployed in different areas and each sensor sends the collected information to the server [12].

Tapiwa M. Chiwewe and Jeofrey Ditsela used IoT and machine learning techniques to create models to predict ground-level ozone levels at specific locations as data sets spanning several years are collected from monitoring stations, the results obtained with prediction accuracy ranging from fair to good [13].

Suganya, E. \& Vijayashaarathi,S. by using PIC microcontroller, WSN and Zigbee found solutions to difficult problems by implementing the internet / intranet on smart vehicles monitoring system for air pollution detection using WSN to measure NO2, humidity, temperature, $\mathrm{CO} 2$ using the sensor of each type 
respectively also the electric circuit has a cloud, wi- fi, GPRS, LCD , manet (Mobile Ad Hoc Network) and routing algorithm. Main system advantage is that it achieves better analysis of mobile vehicles, it can store measured gas values and vehicle details, and an android application can be created to provide an alert message for smartphones [14].

Kan Zheng and others suggested an air quality monitoring system based on LPWA networks which depended on (IoT) technologies that can use emerging machine to machine communication technology (M2M). System benefits are that the LPWA network can provide ranges of over $20 \mathrm{~km}$, LPWA also enables devices to work with long battery life (more than ten years) or solar. The practical circuit consists of: sensor, MCU, battery, the functional components of the monitoring node are shown below in Figure (3) [15].

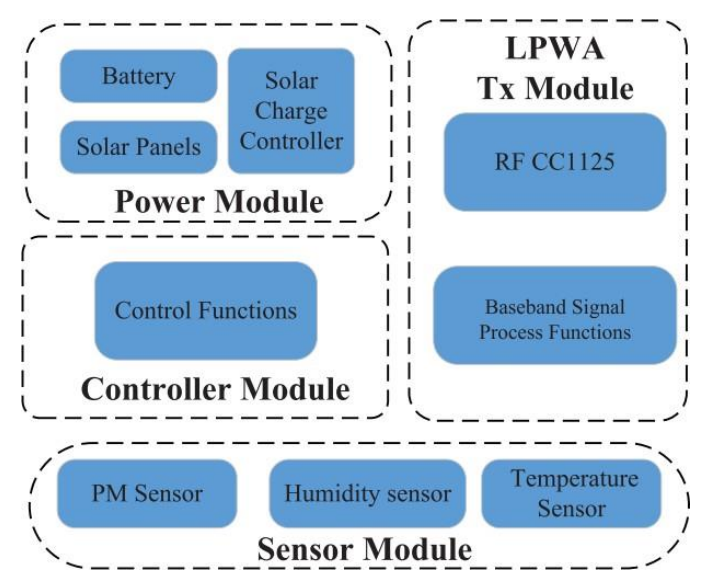

Figure. (3) main functional components of the monitoring node

Somansh Kumar and Ashish Jasuja used Raspberry Pi for real - time air quality monitoring. The system consists of MQ9 to measure CO, MO135 to measure $\mathrm{CO}_{2}$, humidity sensor, temperature sensor, pressure sensor DSM501A which is a PM sensor used for particulate matter monitoring, Arduino Uno and cloud. Raspberry pi is considered as the main node of the system which includes an analog to digital converter (ADC). The data is transmitted using the raspberry Pi through the internet to the cloud. Data stored on the cloud can be restored at any time. System advantage is that compatibility between accuracy and cost was done by making a single board minicomputer Raspberry pi with the system sensors [16].

Gualtier, $\mathrm{G}$ and others introduced an integrated monitoring platform (IMP) system to monitor traffic flows and air pollution real - time and it was tested by installing the sensors at the site of the road where a one-month measurement campaign was carried out the system consists of many practical circuits as follow:

1- Air quality monitoring system using arduino with $\mathrm{CO}, \mathrm{CO} 2$, and $\mathrm{NO}_{2}$ sensors.

2- Traffic monitoring using camera sensors and video analysis software to monitor vehicles

3- Data infrastructure that includes GIS engine, web interface, data storage and management to measure wind direction, temperature and humidity [17].

Parmar, $G$ and others to obtain a fast response, low-cost, low-maintenance system to monitor temperature, humidity and light intensity using WSN to monitor the air quality from inside , and outside proposed architecture consists of three layers the first is sensing layer uses a series of MQ sensors. MQ7 is acarbon monoxide sensor and MQ135 detects NH3, CO2, the second is a network layer which used ESP8266, and finally application layer used Raspberry pi. For displaying data on the website, the MEAN stack was used in [18]

Anwar AL Shamsi and others designed a complete smart system. The system uses a smart air pollution control service aimed at monitoring air pollution. AL Shamsi Smart wsn gas sensor units Microcontroller connected to XBEE (IEEE 802.15.4 Zigbee protocol) analog to digital converter (ADC, the researcher chose a Wasp mote microcontroller because it has a longer life compared to the arduino it is designed for different combinations of connections such as Zigbee and 802.15.4. Unlike the arduino, Wasp mote can accommodate more sensors the temperature, air, and pressure were monitored in addition to carbon dioxide and other pollutants. The pollutants were divided into two groups:

1-(AP I) which contains $\mathrm{C} 6 \mathrm{H} 5 \mathrm{CH} 3, \mathrm{H} 2 \mathrm{~S}$, $\mathrm{CH} 3 \mathrm{CH} 2 \mathrm{OH}, \mathrm{NH} 3, \mathrm{H} 2$, and Air

2- (AP II), which contains $\mathrm{C} 4 \mathrm{H} 10, \mathrm{CH} 3 \mathrm{CH} 2 \mathrm{OH}$, $\mathrm{H} 2, \mathrm{CO}$, and $\mathrm{CH} 4$. [19].

M.F.M Firdhous and others suggested IoT-based indoor air quality monitoring system to track ozone concentrations near the camera, it can send alerts when the pollution level overrides the preset threshold value. The sensor node is designed using an Arduino BT prototype board, and includes an $\mathrm{O} 3$ semiconductor sensor, a small amplifier, a microcontroller (ATmega328P) and a WT11 Bluetooth module [20].

Gagan Parmar and others suggested a system that used Low-cost air quality monitoring nodes and semiconductor gas sensors with Wi-Fi modules. It measures the concentrations of gases 
such as $\mathrm{CO}, \mathrm{CO} 2, \mathrm{SO} 2$, and $\mathrm{NO} 2$. System advantages: low - cost pollution control system, fast response, low maintenance, and production capacity, continuous measurements, small-sized wireless sensor gateway node and database server , and a web server all packed in one package [21].

Mandeep Kumar and others designed a system to monitor five different gases using IoT. The system detects the gas concentration and sends the data to the Thing Speak Storage Cloud. it contains two kinds of sensors, one is calibrated and the other is not calibrated. The system is installed on the wasp mote board with 3G / GPRS network. The data is sensed then sent to the cloud storage for future analysis

The practical circuit consists of the following:

1- Calibrate sensors, include: SO2-A4 sensor for measuring sulfur dioxide (SO2), 4-PH3-20 Sensor for measuring phosphine (PH3) gas, 4-H2- 1000 Sensor for hydrogen measurement

2- Non-calibrated sensors, include: MiCS-2714 Sensor for measuring nitrogen dioxide (NO2) TGS2444 Sensor for ammonia (NH3)

3- Sensor Boards [22].

Candia and others Introduced the urban air quality monitoring system experience relies on the Lora WAN network that uses free environments for hardware and software. Artichecture of the network is deployed in a star- of-stars topology. The researchers tested three Nova SDS011 sensors Nova SDS021y SHINYEI PPD42 interconnected with MiniVol TAS Air metrics the system explain the basis of Lora Wans based on WSN with sensors to improve early warning systems for measuring air quality by remote sensing technology, but Since the researcher used the low cost of the equipment, the challenge is its strength and degredation over time, and this requires periodic [23].

Dhruvil Shah and others used IoT technology for air and sound pollution monitoring. They measured the intensity of noise and gases such as carbon monoxide, smoke, cooking fumes and carbon dioxide are monitored in real time. System advantage because the use of sensors cooperating with the Internet can cause pollution, less complex monitoring, less time- consuming and flexibility, sensors also have a long life, are readily available, less expensive, easy to handle and compact [24].

Kavitha.B.C and others proposed a design that can be used to monitor a specific area and measure air quality. Toxic gases, such as carbon monoxide (CO), liquefied petroleum gas (LPG), methane, butane, and air quality, and then obtaining sensor data collected and uploaded to Google, making it easier to monitor from any part of the world is based on IoT. The air quality can be displayed on the lcd screen and the alarm can also be triggered when the air quality drops to a certain level.The practical circuit consists of: 3 main sensors are MQ-6, MQ-7, MQ-135, DHT11 (temperature and humidity) sensor, Raspberry - pi, lcd, IoT shield [25].

Pradeep D. Landge and R.R. Harne designed a model that starts with sensors that can sense, calculate, and connect data in the network. It measures PM2.5, temperature, humidity, then the monitored data was transmitted wirelessly through Wi-Fi to the server. The workable circuit consisted of sensors for carbon monoxide (CO), carbon dioxide (CO2), nitrogen dioxide (NO2), methane (CH4), hydrogen sulfide (H2S), ammonia (NH3), and particulate matter (PM). ESP8266, AT Command Library [26].

Harsh N. Shah and monitored air quality by using IoT based monitoring. Disadvantages of conventional monitoring: the tools are large, heavy, and expensive. The block diagram is shown in Figure (4) consists of:

1- Hardware: MQ135 gas sensor, Arduino Uno, wi-fi module ESP8266

$16 \times 2 \mathrm{lcd}$, breadboard, $10 \mathrm{~K}$ potentiometer, 220 -

ohm resistor, buzzer

MQ 6 lpg, temperature LM35, humidity SYH5220 sensors

2- Arduino 1.6.13 Software and C Language.

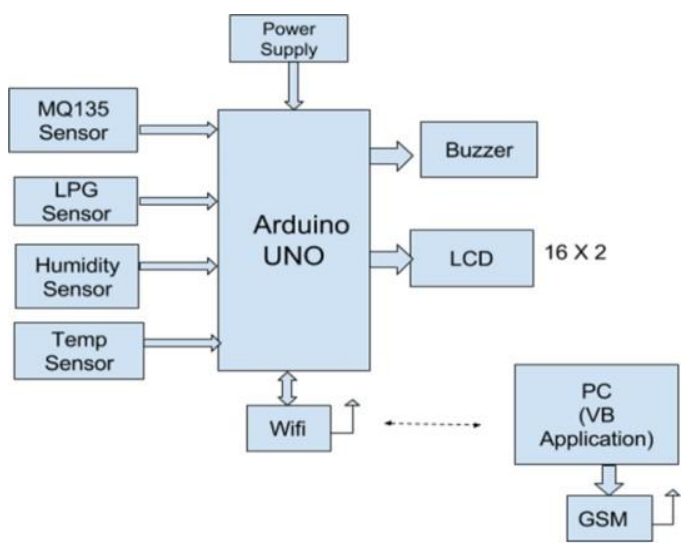

Figure (4) Block diagram of the practical

circuit Advantages of the system:

- 1) Easy installation

2) Directly updates to the mobile.

3) Pollution control

4) Site remotely monitoring [27]. 
Anindya Ananda Hapsari and others analyzed and summarized articles about IAQ using IoT obtained from three databases. The system uses sensors protocols and IoT against the IAQ. The MQ, DHT, SHT gas sensor is a kind of cheap sensor that is often used and it can be an option for the built-in IAQ monitoring system [28].

Jia Uddin and others implemented a set of Android and server applications and gas sensors $\left(\mathrm{CO}_{2}, \mathrm{CO}, \mathrm{LPG}\right.$, and $\left.\mathrm{CH}_{4}\right)$ to sense the air quality in the environment and show the true condition of the air. The proposed model of the system is shown in Figure (5).

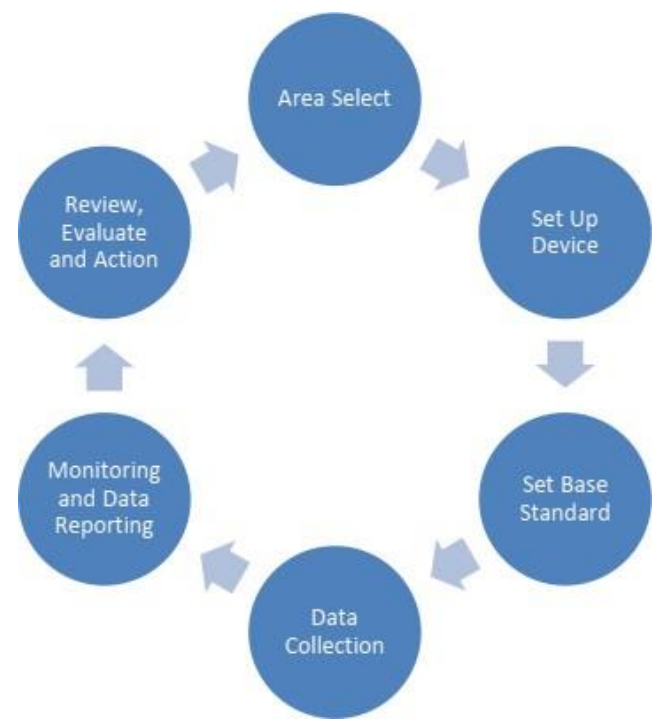

Figure (5) proposed a model of the system

This device consists of the following components: 1.Raspberry Pi 3400

1. DHT 11 Temperature and Humidity sensor

2. MQ-2 Smoke / LPG / CO Gas Sensor

3. M135 Air quality sensor module

4. MQ-8 Hydrogen gas sensor module

5. Sound sensor module

6. TP4056 Charger Module

8. 3.7V $3800 \mathrm{mAh}$

9. Android charger

10. Potentiometer / Buck Booster

11. Wire

12. Breadboard

13. Push -buttons

14. LED [29].

Ashish Gupta and Rajesh Kumar made research that develops a portable, low-cost, and effective pollution monitoring device. MQ series semiconductor sensors used. several traditional emissions control methods adopted:

1. Estimating fossil fuels and accounting for the consumption of raw materials

2. Measuring the flow of carbon dioxide in the air using infrared radiation Wireless sensor node development and deployment of wireless sensor networks based on coverage area and scalability.

Hardware requirements

1. Arduino Yun suitable microcontroller

2. An array of sensors to read the gaseous concentration in the environment.

3. Programming environment or IDE that would be used

4. Box / housing for the whole system [30].

Cynthia, J and others suggested monitoring air pollution levels from various pollutants Pollution will be measured by selecting three Geographic areas: resident, industrial area and traffic areas, the measurement will be done by IoT , and store the measurements in the cloud, then we will analyze and measure the level of pollution in any particular place using WSN they record the measurements for five consecutive days and store them in the cloud for use. The electric circuit as shown in Figure (6) consisted of $\mathrm{NO}_{\mathrm{X}}$ sensor, $\mathrm{CO}$ sensor, $\mathrm{SO}_{2}$ sensor, humidity sensor, heat sensor, Raspberry PI, GPS. For $\mathrm{NO}_{2}$ authors submitted a relationship between temperature and resistance as in equation (1):

$$
\begin{aligned}
& \mathrm{R}_{\mathrm{T}}=\mathrm{R}_{\mathrm{b}} \alpha \exp (\mathrm{T}) \\
& \mathrm{R}: \quad \text { resistance, } \mathrm{T}: \text { temperature } \\
& \mathrm{R}_{\mathrm{b}} \text { : constant, } \alpha \text { :Direct proportion } \\
& \text { The relationship between humidity and } \\
& \text { resistance is presented in equation (2): } \\
& \mathrm{R}_{\mathrm{air}}=\mathrm{cR}_{\mathrm{t}} \mathrm{H}_{\mathrm{d}}
\end{aligned}
$$

$\mathrm{R}_{\mathrm{t}}$ : room temperature, $\mathrm{H}$ : humidity

The data acquisition unit consists of hardware sensors which together measure air quality index (AQI) which is used to measure pollution levels in ambient air and can be calculated as in equation (3) as follows:

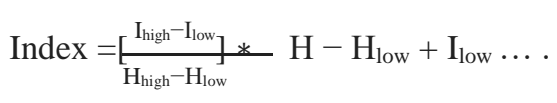

where:

Index $=$ (Air Quality) index

$\mathrm{H}=$ pollutant cocentration

$\mathrm{H}_{\text {low }}=$ concentration breakpoint that is $\leq \mathrm{H}$

$\mathrm{H}_{\text {high }}=$ concentration break point that is $\geq \mathrm{H}$

$\mathrm{I}_{\text {low }}=$ index break point corresponding to

$\mathrm{H}_{\text {low }}$

$\mathrm{I}_{\text {high }}=$ index break point corresponding to $\mathrm{H}_{\text {high }}$ [31]. 


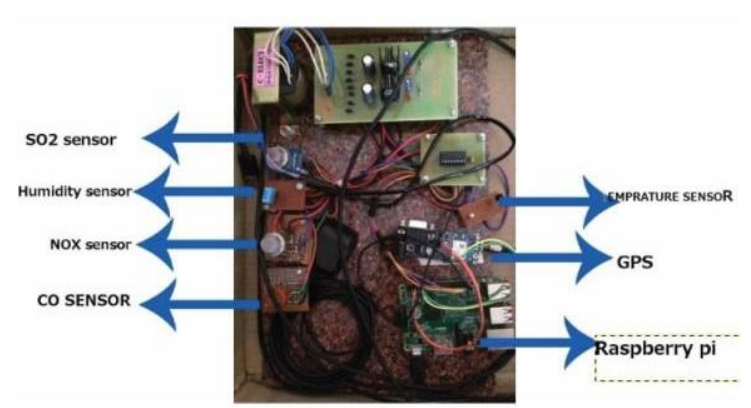

Figure (6) the electric circuit

Kinnera Bharath Kumar Sai and others talked about air pollution from transportation, and deforestation. The measurement will be performed by using MQ sensors suitable for installation inside or outside the building or home, where MQ135 is used to detect ammonia, carbon dioxide, alcohol, smoke, and MQ7 is used to detect carbon monoxide. The practical circuit consists of Arduino uno, ESP8266, LCD, DHT11 temperature , and humidity sensor [32].

Vladimir Shakhov \& Olga Sokolova suggested a wireless network with mobile for moving objects (vehicles), taking into account two variables of road traffic, which are deterministic and poison flow. One of the advantages of the system is the use of WSN as it is characterized by energy independence of the nodes, the ability to make flexible changes in the network topology, improved spatial-temporal details of measurements, lower operating costs, and also the system's features that the movement of sensors can lead to increased monitoring efficiency [33].

Zhiwen $\mathrm{Hu}$ and others designed a system that offers the accuracy and real - time of energysaving on aerial and ground sensing system. The system architecture consists of a sensor layer for data collection, a transmission layer to enable twoway communications, a processing layer for data analysis and manipulation, and a presentation layer to provide a graphical interface to users. System disadvantages: at the sensor layer, sensor and data download are not expected continuously due to limited battery capacity [34].

Vijayakumar Sajjan, Pramod Sharma designed IoT-based air pollution monitoring framework .The MQ series sensor is connected to a Node MCU equipped with an ESP8266 WLAN connector server to send the sensor to a coil and the cloud. Previous works include:

1- Zigbee wireless air pollution monitoring with low cost and energy - efficient sensors.

2- Pollution control system using WSN

3- WSN based on air pollution monitoring system in major cities [35]

Nael Abd Alfatah Husein and others proposed a wireless device that has two significant sides: energy , and cost, they designed a system for data monitoring, and air pollution analysis in indoor and outdoor environments. The advantages of the proposed system are the low- cost, small size, long coverage and real - time transmitting [36].

Gupta, $\mathrm{H}$ and others suggested that pollution can be monitored by determining the air quality index (AQI), as the continuous check for remote air pollution uses framework XBEE despite using ZigBee and GPS framework based on WSN, but $\mathrm{XBEE}$ is used to send data to the computer as the system consists of a series of MQ sensors for measuring gases, Node MCU, ESP8266 wlan for cloud sending, GPS [37].

Aarathi Ramesh Muppalla and others introduced an all-in-one solution for low-cost air pollutant monitoring. Any IoT solution contains three components:

1- Sensors: in this project LORAWAN network was used, $\mathrm{CO}, \mathrm{CO} 2, \mathrm{PM} 2.5$ sensors were used.

2- Network server: which is used to gather, process and store data from sensors.

3- Web server: for this design NIGEX is an open - source high - performance HTTP server, Kibana dashboards are reverse proxied through NIGEX server.

where the data is gathered from the $\mathrm{CO}, \mathrm{CO} 2$, PM2.5 sensors every five seconds and it is stored in the Elasticsearch database [38].

Barot and others suggested an air quality monitoring system that is based on IoT technology.It is used to measure indoor, and outdoor air pollutants, the system will measure PM10, PM2.5, carbon monoxide, temperature and humidity ,it consisted of an air quality sensor node controller connected to a public cloud medium (MQTT) (Message Queue Telemetry Transport protocol)ESP8266 is equipped with $\mathrm{Wi}-\mathrm{Fi}$ to smooth MQTT protocol ,system also contained DHT22 sensor, electrochemical sensor ZE07-CO for carbon monoxide SDS021 sensor unit for PM10 and PM2.5 readings .The system advantages are: by using MQTT which is an ISO - based open source messaging protocol file that works on the TCP / IP network layer, it provided connections to remote sites the system also reduced power consumption by converting a smart sensor node 
into five different modes, the topology design is shown in Figurer (7) [39].

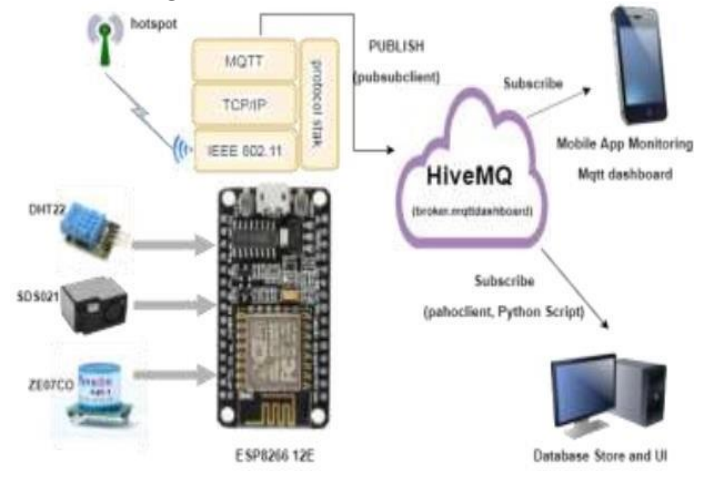

Figure (7) topology design

Ajitesh Kumar and others developed a portable air quality monitoring system that enabled real-time data analysis. Three sensors are used : MQ9 gas sensor which senses carbon monoxide, MQ2 sensor which senses smoke, and PMS3003 G3 particle sensor for PM2.5 give readings to the Node MCU as a processing unit and send this detection data on the Internet, to store and restore data via Hypertext Transfer Protocol (HTTP) over the Internet. The circuit included :bread board, PMS3003G3 particle sensor, connecting wires, 180 and 330-ohm resistor,MQ9,MQ2gas sensors,3.3 $5 \mathrm{v}$ boost converter, analog multiplexer 4051, OLED display, Battery Charger Board for Li-ion battery Node MCU 1.0 ESP8266.

System advantages: It is portable, affordable, takes less space, can be installed anywhere and provides operational efficiency and flexibility than traditional wired methods it also supports the newest technologies like Node, Ruby, etc. [40].

Satyam Yadav and others designed a system used gas sensors and is sensitive and fast to detect toxic gases present in the surrounding areas such as carbon dioxide, smoke, gasoline, NH3, nitrogen oxides. Operation circuit: MQ6 LPG sensor. The MQ135 dynamic gas sensor is used which can sense NH3, NOx, gasoline, smoke, and carbon dioxide. Arduino controller. Table (1) shown below discusses the differences between traditional and IoT-based methods of monitoring air quality.
Table (1)

differences between traditional and IoTbased methods of monitoring air quality.

\begin{tabular}{|l|l|l|}
\hline$\underline{\text { Parameter }}$ & Conventional & $\underline{\text { IOT APM }}$ \\
\hline$\underline{\text { Accuracy }}$ & $\underline{\text { High }}$ & $\underline{\text { Low }}$ \\
\hline$\underline{\text { Coverage }}$ & $\underline{\text { Low spatiotemporal }}$ & $\underline{\text { High }}$ \\
\hline$\underline{\text { Detection }}$ & $\underline{\text { Large scale }}$ & $\underline{\text { Small scale }}$ \\
\hline$\underline{\text { Cost }}$ & $\underline{\text { High }}$ & $\underline{\text { Cheap }}$ \\
\hline $\begin{array}{l}\text { Power } \\
\text { consumption }\end{array}$ & $\underline{\text { High }}$ & Low \\
\hline
\end{tabular}

The disadvantages of traditional monitors: their large size, heavyweight and high cost [41].

Ms. Monika Patil and others suggested a solution for monitoring air and noise pollution levels in an industry environment or by using an embedded wireless computing system. This model is adaptable and deployable to any infrastructure environment. Practical circuit: Arduino Nano, humidity, temperature and dust levels sensors, Raspberry. Wi-Fi module and temperature, humidity, gas and dust sensors are connected with Arduino [42].

Vaibhav Dinkar Shinde proposed that we can use IoT technology to obtain data from sensors for toxic gases such as carbon dioxide and sulfur dioxide to calculate pollution we use a sensor that senses harmful gases and then a sensor generates data and transmits this data to users, the practical circuit lcd, MQ2 is an air sensor:

MQ135 gas sensor, Arduino Uno, Wi-Fi module ESP8266, 16X2 lcd, breadboard, 10K potentiometer, $1 \mathrm{~K}$ ohm resistors, $220 \mathrm{ohm}$ resistor, buzzer [43].

Mehmet Taştan proposed the AAL Ambient Assisted Living System for helping the elderly and children especially to live safely in their homes it gives real-time monitoring of indoor air quality parameters like $\mathrm{CO} 2, \mathrm{CO}, \mathrm{PM} 10, \mathrm{NO} 2$, temperature , and humidity through a mobile user interface developed with the Blynk platform IoT. The practical circuit consists of an ESP32 and a set of air quality sensors. Detection unit includes GP2Y1010AU, MH-Z14, MICS4514 and DHT22 sensors. Advantages are low - cost, open - source technology, ease of installation, portability, and easy configuration. System disadvantages: scalability, quality of services, security, and privacy [44].

M. Babykala and others designed a project the main objective of it is to control air pollution 
by designing and implementing an air quality monitoring (AQM) system to reduce air pollution. Operation circuit: led display, temperature humidity sensor, carbon monoxide sensor, low concentration ozone sensor, grove particle sensor [45].

\section{Tools that are used in smart environment monitoring system}

Tools are divided into two parts:

A- Microcontrollers as follows: ArduinoUNO: Arduino Uno as shown in Figure (8) is a microcontroller board based on the ATmega328P.It contains 14 digital input/output pins 6 analogy inputs, a $16 \mathrm{MHz}$ quartz crystal, a USB connection, a power jack, an ICSP header, and a reset button [46].

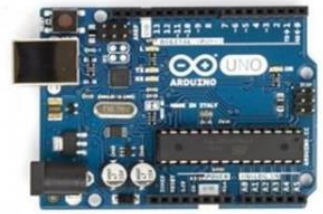

Figure (8) ArduinoUNO

WIFI Module (ESP8266): -

The ESP8266 as shown in Figure (9) is a low-cost Wi-Fi chip with TCP/IP stack and MCU capability. It works on $3.3 \mathrm{~V}$ and gives the system access to Wi-Fi [47].

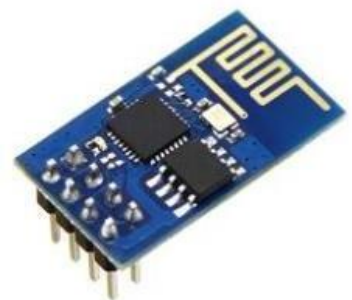

Figure (9) WIFI Module (ESP8266)

Raspberry pi: -

The Raspberry Pi is a low-cost single-board computer it is fully supported by Simulink; and it can be programmed according to the design paradigm, which means that anyone can design and implement applications on Raspberry Pi boards which is shown in Figure (10)[48].

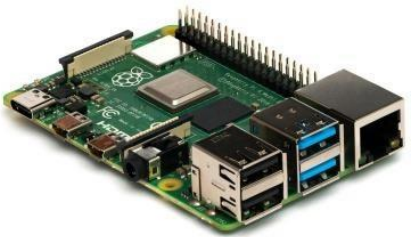

Fig (10) Raspberry Pi board

B- Sensors as follows:

1- Humidity and temperature sensor:

This sensor shown below in Figure (11) can be easily interfaced with any microcontroller such as Arduino, Raspberry Pi etc... to measure humidity and temperature instantaneously [29].

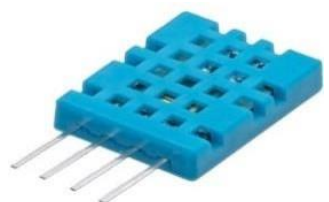

Figure (11) Humidity and temperature sensor

2- CO sensor

Carbon monoxide gas is a simple molecule: one-part carbon and one-part oxygen. Carbon monoxide is hard to detect without a sensor, which is one of the reasons it's so dangerous. $\mathrm{CO}$ sensor is shown in Figure (12) [49].

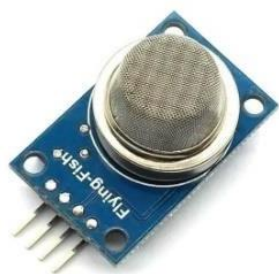

Figure (12) CO sensor

3- $\mathrm{CO}_{2}$ sensor:

A carbon dioxide $\left(\mathrm{CO}_{2}\right)$ sensor shown in Figure (13) below is a small instrument that can detect and measure carbon dioxide within the surrounding air [50].

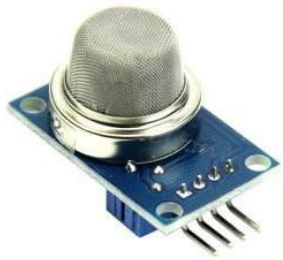

Figure (13) $\mathrm{CO}_{2}$ sensor 
4- $\mathrm{NO}_{\mathrm{x}}$ sensor

It is typically a high- temperature device built to discover nitrogen oxides. The term NOx represents many forms of nitrogen oxides like $\mathrm{NO}$ (nitric oxide), $\mathrm{NO}_{2}$ (nitrogen dioxide) and $\mathrm{N}_{2} \mathrm{O}$ (nitrous oxide, or laughing gas). The sensor is shown in Figure (14).[51]

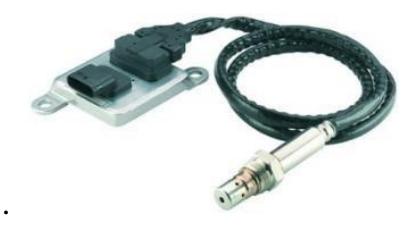

Figure (14) $\mathrm{NO}_{\mathrm{x}}$ sensor

5- Intensity Illumination sensor: is a digital ambient light sensor is possible to detect a wide range at high resolution. It is shown in Figure. (15). [52]

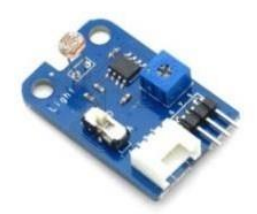

Figure (15) Intensity Illumination sensor

6- $\mathrm{SO}_{2}$ sensor

$\mathrm{SO} 2$ in ambient air is monitored by several methods and instruments such as UV or IR spectroscopy and solid-state electrochemistry techniques. $\mathrm{SO}_{2}$ sensor is shown in Figure (16) below [53]

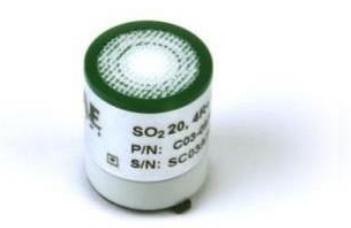

Figure (16) $\mathrm{SO}_{2}$ sensor

7- MQ135 sensor:

It has sensibility to NH3, NOx, alcohol, Benzene, smoke, $\mathrm{CO} 2$ and some other gases. Its output in form of voltage levels [46] has many features such as High sensitivity, Fast response,Wide detection range, Stable performance and long life, simple drive circuit, it is shown in Figure (17).[54]

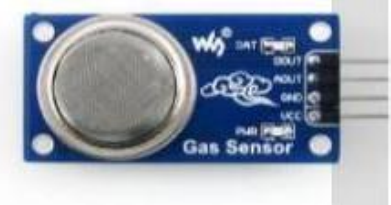

Figure (17) MQ135 sensor

\section{Conclusion:}

IoT technology is used for air quality monitoring issues, giving real - time data and fast response.

These systems monitor the ambient air of the environment using different tools, the most common microcontroller used is an Arduino microcontroller. The device collects the data of the investigating site at every second and transmits data via Wi-Fi and notify the organization about whether air is good, poor or dangerous and notify about the threshold level. This prototype can be carried as a low cost mobile device that anyone can use and it monitors the air quality around themselves and gets assured whether they are inhaling polluted or fresh air, and what they can avoid, not to contribute to polluting the air. IoT based air pollution monitoring technology uses sensors which are easily available and detects the wide range of harmful gases like $\mathrm{NO} 2, \mathrm{CO} 2, \mathrm{CO}$ etc. It gives fast response, real time data and is having long lifetime. The most common sensor used is $\mathrm{CO}_{2}$ sensor.

\section{Future Work:}

The project can be improved by:

1-Using a solar cell instead of a battery. Current estimates show that by the year 2025, the world is likely to have roughly 75 billion devices connected via the Internet of Things (IoT), most of that are probably going to be located inside. Given the eventual broad deployment of such devices, supplying them with power by way of batteries or connection to the grid will have to be replaced by local energy sources that require little to no maintenance. Because of artificial intelligence and automated learning, the cell system is now capable of tailoring computational workloads according to the level of illumination, which allows it to reduce energy consumption and battery waste

2-Using particle PHOTON instead of other microcontrollers. PHOTON is a tiny $\mathrm{Wi}-\mathrm{Fi}$ IoT device for creating connected projects and products for the Internet of Things. The board 
itself uses a Cypress Wi-Fi chip (one that can be found in Nest Protect, LIFX, and Amazon Dash) alongside a powerful STM32 ARM Cortex M3 microcontroller. It's like the Spark Core. Has many benefits such as:

A complete edge-to-cloud platform for our business

Bridge the gap between the physical and digital world

A customize tracking system for our needs.

\section{References:}

[1] R. Xu, Q. Zeng, L. Zhu, H. Chi, X. Du, and M. Guizani, "Privacy leakage in smart homes and its mitigation: Ifttt as a case study," IEEE Access, vol. 7, pp. 63457-63471, 2019.

[2] M.Yun, \& B.Yuxin, "Research on the architecture and key technology of Internet of Thing (IoT) applied on smart grid". 2010 International Conference on Advances in Energy Engineering, 2010. IEEE, 69-72, 2017.

[3] Z.Zhu, \& R.-G.Huang, "Study on the IoT architecture and access technology". 16th International Symposium on Distributed Computing and Applications to Business, Engineering and Science (DCABES), IEEE, 113116, 2017.

[4] A. K. Waleed, P. D. Kusuma, and C. Setianingsih , "Monitoring and Classification System of River Water Pollution Conditions with Fuzzy Logic," IEEE International Conference on Industry 4.0, Artificial Intelligence, and (IAICT IEEE, pp. 112-117, ), 2019. Communications Technology, 2019.

[5] P. M. Sebastian, C. Romeo, and U. GeorgeAndrei, "Environmental Pollution Control," in 2019 International Conference on Electromechanical and Energy Systems (SIELMEN), IEEE, pp. 1-5, 2019

[6]M. A. Matin and M. Islam, "Overview of wireless sensor network," Wireless Sensor Networks-Technology and Protocols, pp. 1-3, 2012

[7] S. Devarakonda, P. Sevusu, H. Liu, R. Liu, L. Iftode, and B. Nath, "Real-time air quality monitoring through mobile sensing in metropolitan areas," in Proceedings of the 2nd
(ACM SIGKDD )international workshop on urban computing, pp. 1-8, 2013.

[8] S.Manna, S. S.Bhunia, \& N.Mukherjee, "Vehicular pollution monitoring using IoT. International Conference on Recent Advances and Innovations in Engineering" (ICRAIE-2014) IEEE, 1-5, 2014.

[9] S.Shailendra J.Rajeev, R.Priya and R. T.Malay "Software aspects of WSN for monitoring in an Indian Greenhouse", International Conference on Computational Intelligence and Communication Networks (CICN), IEEE, pp 168-172, 2015.

[10] M. S.Jamil, M. A.Jamil, A.Mazhar, A.Ikram, A.Ahmed, \& U.Munawar, "Smart environment monitoring system by employing wireless sensor networks on vehicles for pollution free smart cities". Procedia Engineering, 107, 480-484, 2015.

[11] S.Bedoui, S.Gomri, H.Samet, \& A.Kachouri, "Air pollution monitoring system using LabVIEW". 2015 IEEE 12th International Multi-Conference on Systems, Signals \& Devices (SSD15), IEEE, 1-6,2015.

[12] S. Sirsikar and P. Karemore, "Review paper on air pollution monitoring system," International journal of Advanced research in Computer and Communication Engineering, vol. 4, no. 1, pp. 218-20, 2015.

[13] T.M.Chiwewe, and D.Jeofrey "Machine learningbased estimation of Ozone using spatiotemporal data fromairquality monitoring stations." IEEE 14th International ConferenceInIndustrial Informatics (INDIN),pp. 58-63, 2016

[14] E.Suganya, \& S.Vijayashaarathi, "Smart vehicle monitoring system for air pollution detection using WSN". International Conference on Communication and Signal Processing (ICCSP), IEEE, 0719-0722, 2016.

[15] K. Zheng, S. Zhao, Z. Yang, X. Xiong, and W. Xiang, "Design and implementation of LPWA-based air quality monitoring system," IEEE Access, vol. 4, pp. 3238-3245, 2016. 
[16] S. Kumar, \& A.Jasuja, "Air quality monitoring system based on IoT using Raspberry Pi'.International Conference on Computing, Communication and Automation (ICCCA), IEEE, 1341-1346, 2017.

[17] G.Gualtieri, F.Camilli, A.Cavaliere, T.De Fillippis, D. Gennaro, F. Di Lonardo, S. Dini, F., B.Gioli, Matese, A. \& W. Nunziati, "An integrated low-cost road traffic and air pollution monitoring", 2017.

[18] G., Parmar, S.Lakhani, \& M. K.Chattopadhyay, "An IoT based low cost air pollution monitoring system". International Conference on Recent Innovations in Signal processing and Embedded Systems (RISE), IEEE, 524-528, 2017.

[19] A., Alshamsi, Y., Anwar, M., Almulla, M., Aldohoori, N.Hamad, \& M.Awad, "Monitoring pollution: Applying IoT to create a smart environment". International Conference on Electrical and Computing Technologies and Applications (ICECTA), IEEE, 1-4, 2017.

[20] M. Firdhous, B. Sudantha, and P. arunaratne, "IoT enabled proactive indoor air quality monitoring system for sustainable health management," 2nd International Conference on Computing and Communications Technologies (ICCCT), IEEE, pp. 216-221, 2017.

[21] L.S.Parmar, and M. K. Chattopadhyay, "An IoT based low cost air pollution monitoring system," in 2017 International Conference on Recent Innovations in Signal processing and Embedded Systems (RISE) IEEE, pp. 524-528, 2017.

[22] M.Kumar, S.Mini, \& T.Panigrahi, "A scalable approach to monitoring air pollution using IoT". 2nd International Conference on I-SMAC (IoT in Social, Mobile, Analytics and Cloud)(ISMAC) I-SMAC (IoT in Social, Mobile, Analytics and Cloud)(I-SMAC), 2018 2nd International Conference IEEE, 42-47, 2018.

[23] A., Candia, S. N.Represa, D.Giuliani, M. Á.Luengo, A.A.Porta, \& L.A.Marrone, "Solutions for SmartCities: proposal of a monitoring system of air quality based on a LoRaWAN network with low-cost sensors". 2018 Congreso Argentino de Ciencias de la Informática y Desarrollos de Investigación (CACIDI) IEEE, 1-6, 2018.

[24] D. Shah, P. Kudale, and S. J. Prasad Shirwadkar, "Iot Based Air and Sound Pollution Supervising System," IOSR Journal of Engineering, 2018.

[25] D. Jose, "IoT Based Pollution Monitoring System Using Raspberry-Pi," International Journal of Pure and Applied Mathematics, vol. 118, no. 24, 2018.

[26] P. D. Landge and R. Harne, "Air Quality Monitoring System for City: A Review," Int. Res. J. Eng. Technol, vol. 5, no. 1, pp. 5-6, 2018.

[27]H. N. Shah, Z. Khan, A. A. Merchant, M. Moghal, A. Shaikh, and P. Rane, "IoT based air pollution monitoring system," International Journal of Scientific \& Engineering Research, vol. 9, no. 2, pp. 62-66, 2018.

[28] A. A. Hapsari, A. I. Hajamydeen, and M. Abdullah, "A review on indoor air quality monitoring using IoT at campus environment," International Journal of Engineering Technology, vol. 7, no. 4.22, pp. 55-60, 2018.

[29] M. Khan, H. B. Hossain, R. U. Rahman, M. Arafat, and M. Jony, "Air pollution monitoring system for smart city," BRAC Univeristy, 2018.

[30] A. Gupta and R. Kumar, "An IoT Enabled Air Quality Measurement," Indian Journal of Science and Technology, vol. 11, p. 46, 2018.

[31] J.Cynthia, M.Saroja, P. Sultana, \& J. Senthil, "IoT-Based Real Time Air Pollution Monitoring System". International Journal of Grid and High Performance Computing (IJGHPC), 11, 28-41, 2019.

[32] K. B. K.Sai, S.Mukherjee, \& H. P. Sultana, "Low Cost IoT Based Air Quality Monitoring Setup Using Arduino and MQ Series Sensors With Dataset Analysis". Procedia Computer Science, 165, 322-327, 2019.

[33]V. Shakhov and O. Sokolova, "Towards Air Pollution Detection With Internet of Vehicles," in 2019 15th International Asian School-Seminar Optimization Problems of Complex Systems (OPCS), IEEE, pp. 183-186, 2019. 
[34] Z. Hu, Z. Bai, Y. Yang, Z. Zheng, K. Bian, and L. Song, "UAV aided aerial-ground IoT for air quality sensing in smart city: architecture, technologies, and implementation," IEEE Network, vol. 33, no. 2, pp. 14-22, 2019.

[35] V. Sajjan, P.Sharma "Research on an Iot Based Air Pollution Monitoring System" International Journal of Innovative Technology and Exploring Engineering (IJITEE) Volume-8, Issue- 9S2, July 2019

[36] N. A. A. Husein, A. H. Abd Rahman, and D. P. Dahnil, "Evaluation of LoRa-based Air Pollution Monitoring System," IJACSA) International Journal of Advanced Computer Science and Applications, vol. 10, no. 7, 2019.

[37]H.Gupta, D.Bhardwaj, H.Agrawal, V. A. Tikkiwal, \& A. Kumar, "An IoT

Based Air Pollution Monitoring System for Smart Cities". 2019 IEEE International Conference on Sustainable Energy Technologies (ICSET), IEEE, 173-177,2019.

[38]A. R. Muppalla et al., "Design and Implementation of IoT Solution for Air Pollution Monitoring," in 2019 IEEE Recent Advances in Geoscience and Remote Sensing: Technologies, Standards and Applications (TENGARSS), IEEE, pp. 45-48, 2019.

[39] V.Barot, V.Kapadia, \& S.Pandya, "QoS Enabled IoT Based Low Cost Air Quality Monitoring System with Power Consumption Optimization". Cybernetics and Information Technologies, 20, 2020.

[40]A. Kumar, M. Kumari, and H.Gupta, "Design and Analysis of IoT based Air Quality Monitoring System," in 2020 International Conference on Power Electronics \& IoT Applications in Renewable Energy and its Control (PARC): IEEE, pp. 242-245 , 2020

[41] S.Yadav, K.patil, M.Yungpisangle, K L.Poushong "IoT Based Air Quality Monitoring System" International Research Journal of Modernization in Engineering Technology and Science Volume:02/Issue:07/July-2020

[42] M. M. Patil, "Monitoring system of dust \& humidity in environment using Arduino and web of thing ",
IJRAR-International Journal of Research and Analytical Reviews (IJRAR), vol. 7, no. 1, pp. 689692-689-692, 2020

[43] V. Dinkar International Journal of Trend in Scientific Research and Development (IJTSRD) Volume 4 Issue 3, 2020

[44]M. Taştan, "An IoT Based Air Quality Measurement and Warning System for Ambient Assisted Living," Avrupa Bilim ve Teknoloji Dergisi, no. 16, pp. 960-968,2018.

[45]M. Babykala, K. Dineshwaran, C. Gayathri, K. D. Prabu, and S. Akash, "Air Quality Monitoring System."

[46] L. Müller, M. Mohammed, and J. W. Kimball, "Using the Arduino Uno to teach digital control of power electronics," IEEE 16th Workshop on Control and Modeling for Power Electronics (COMPEL), IEEE, pp. 1-8, 2015.

[47] A. Tulenkov, A. Parkhomenko, A. Sokolyanskii, A. Stepanenko, and Y. Zalyubovskiy, "The features of wireless technologies application for Smart house systems," IEEE 4th International Symposium on Wireless Systems within the International Conferences on Intelligent Data Acquisition and Advanced Computing Systems (IDAACS-SWS), IEEE, pp. 1-5, 2018.

[48]A. Nayyar and V. Puri, "Raspberry Pi-a small, powerful, cost effective and efficient form factor computer: a review," International Journal of Advanced Research in Computer Science and Software Engineering, vol. 5, no. 12, pp. 720-737, 2015.

[49] https://edinburghsensors.com/news-andevents/carbon-monoxide-detection-with-a-cosensor/

[50]J. Hodgkinson, R. Smith, W. O. Ho, J. R. Saffell, and R. P. Tatam, "Non-dispersive infrared (NDIR) measurement of carbon dioxide at 4.2 $\mu \mathrm{m}$ in a compact and optically efficient sensor," Sensors and Actuators B: Chemical, vol. 186, pp. 580-588, 2013

[51]Q. Lin and P. Chen, "A simple and effective methodology for estimating urea selective catalytic reduction systems states based on NOx 
sensor measurement," in 2017 American Control Conference (ACC), IEEE, pp. 5367-5372, 2017.

[52] I. Lapyga, "Application of Light Sensors Amplifier and Wireless Networking Sensor for Ambient Light Data to the Android Platform," International Journal of Recent Technology and Engineering (IJRTE), vol. 8, no. 3, 2019.

[53] C. Chatterjee and A .Sen," Sensitive colorimetric sensors for visual detection of carbon dioxide and sulfur dioxide," Journal of Materials Chemistry A, vol. 3, no. 10, pp. 5642-5647, 2015.

[54] https://components101.com/sensors/mq135gas-sensor-for-air-quality.

\section{دراسة استقصائية حول نظام المراقبة الأكي للبيئة القائم على إنترنت الأشياء}

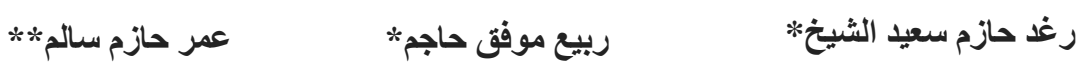

omer.salim@monash.edurabeehagem@uomosul.edu.iq raghad.h.alshekh@uomosul.edu.iq

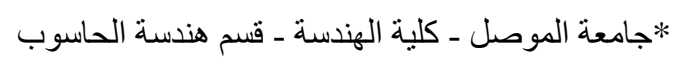

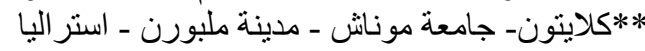

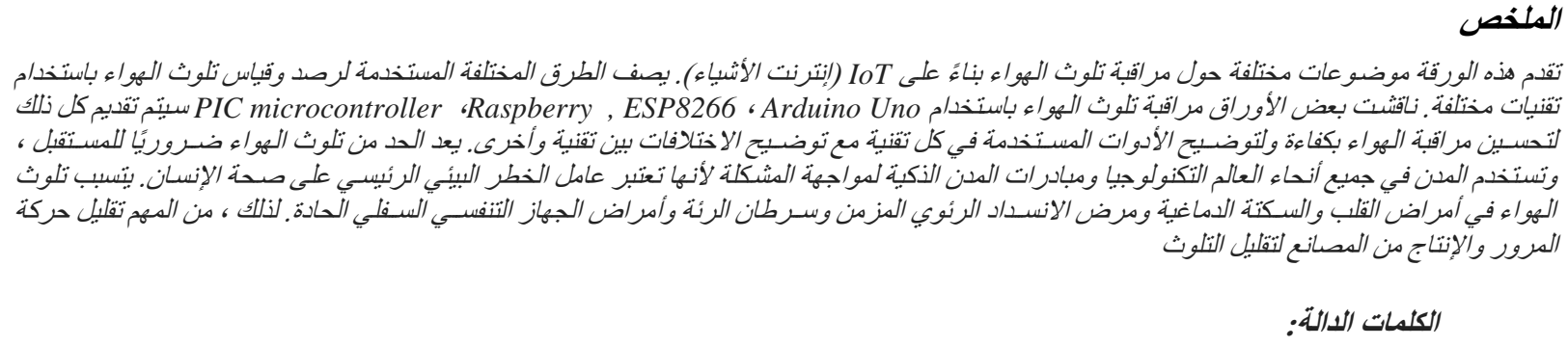

انترنت الأشياء ، تلوث الهواء ، اردوبيو ، Raspberry, ESP8266 ، الدستشعرات ، شبكة استشعار لاسلكية . 